\title{
Mineração
}

\section{Modelo de previsão do custo de mineração pelo sistema de curvas de aprendizado}

\author{
Walmir Carvalho Pereira \\ Eng. Chefe do Departamento de Planejamento de Mina,MSc - Samarco Mineração S.A. \\ E-mail:walmir@samarco.com.br \\ Saul B. Suslick \\ Departamento de Geologia e Recursos Naturais - Instituto de Geociências - UNICAMP \\ E-mail:suslick@ige.unicamp.br
}

\section{Resumo}

Esse trabalho apresenta o desenvolvimento de um modelo de previsão de custo de produção por intermédio da técnica do sistema de curvas de aprendizado. Uma das vantagens desse modelo é a possibilidade de incorporar as mudanças técnico-econômicas ocorridas no desempenho do custo, que se refletem na série temporal em análise. Esse modelo possibilita estimar com relativa precisão o desempenho dos custos atuais e futuros utilizando-se de uma série histórica de dados de produção de uma unidade de mineração de ferro. A comparação entre os custos reais de operação na mina e os resultados gerados pelo modelo de Stanford-B de aprendizado situou-se dentro de uma faixa aceitável de tolerância próxima de $10 \%$.

Palavras-chave: custo de mineração, curvas de aprendizado, minério de ferro e planejamento mineral.

\begin{abstract}
This paper presents a learning curve model for forecast the operational mining cost. One of the great advantages of this model is the possibility to include the technical and economic changes in the cost performance that are incorporated by the time series. The learning model projections presents a good level of accuracy for cost data from iron ore. The comparison between actual and forecast data costs through the Stanford-B model indicates that the output values are within an acceptable range of $10 \%$ confidence interval.
\end{abstract}

Keywords: mining cost, learning curves, iron ore and mining planning. 


\section{Introdução}

Sabe-se que o desempenho melhora com a prática e que esse fenômeno é amplamente observado nas diversas situações industriais. Um meio racional de medir e prever melhorias de produtividade, conhecida como a técnica do sistema de curvas de aprendizado, já está bastante difundido e documentado. A forma mais simples de uma curva de aprendizado expressa o custo unitário acumulado da produção como função da produção acumulada, com a taxa de melhoria constante entre níveis de produção crescentes. O presente trabalho foi desenvolvido com o objetivo de introduzir o sistema de aprendizado como uma técnica de previsão, a partir de um estado predefinido do sistema e da manutenção das condições de trabalho. Para avaliar o desempenho dessa técnica, foram analisadas as várias fases de produção de uma unidade de mineração de ferro, vinculado a um sistema integrado de operações de beneficiamento, transporte de concentrados através de mineroduto, pelotização e porto. Os dados de produção se referem à Gerência de Mineração da SAMARCO e estão distribuídos mensalmente em um período, três anos (1997/2000). Esse período foi escolhido por representar uma parte marcante da história da empresa, pois compreende a fase preparatória para o projeto de expansão da empresa (1997), a fase pós-expansão (1998/1999) e a fase de consolidação da nova capacidade produtiva (1999/2000). A fim de garantir o sigilo das informações, os valores de custo aqui apresentados são custos aparentes, mas que guardam entre si e com as demais variáveis em análise as mesmas relações matemático-estatísticas apresentadas pelos custos reais.

\section{Conceitos e parâmetros das curvas de aprendizado}

Os conceitos teóricos dos modelos de aprendizado podem ser encontrados nos trabalhos do Boston Consulting Group (1970), Belkaoui (1968) e Towill (1990). Na indústria mineral algumas aplicações foram realizadas por Pierson
(1981), Suslick e Harris (1990) e Silva e Suslick (1993). A concepção do sistema de aprendizado baseia-se na noção intuitiva de que o incremento de produtividade é resultante de um aprendizado, sendo assim passível de planejamento e previsão. Em termos formais, o modelo de conhecimento pode ser definido através das seguintes relações que representam simples valores acumulados:

Custo acumulado: $\underset{\mathbf{i}=\mathbf{1}}{\mathbf{i}} \mathbf{\mathbf { t }}=\Sigma \mathbf{C}_{\mathbf{i}}$

Produção acumulada: $\mathbf{P t}=\sum_{\mathbf{j}=\mathbf{1}}^{\mathbf{t}} \mathbf{P}_{\mathbf{j}}$

sendo: $\mathbf{Y}=\mathbf{C}_{\mathbf{t}} / \mathbf{P}_{\mathbf{t}}$

a medida do sistema de aprendizado.

O modelo de curva de aprendizado mais utilizado é o potencial simples, onde a relação entre $\mathbf{C}_{\mathbf{t}}$ e $\mathbf{P}_{\mathbf{t}}$ pode ser expressa por:

$\mathbf{C}_{\mathrm{t}}=\mathbf{A} \mathbf{P}_{\mathrm{t}}^{\mathrm{k}}$

onde $\mathbf{A}$ é o custo requerido para produzir a primeira unidade e $\mathbf{k}$ é uma constante relacionada à inclinação da curva de aprendizado. Definem-se índice de aprendizado IA e índice de progresso IPR a partir das expressões:

$\mathrm{IA}=\mathbf{2}^{\mathrm{k}}$

IPR $=1$ - IA

Aplicando logaritmos à relação (2) tem-se :

$\operatorname{Ln} C_{t}=\operatorname{Ln} A+k \cdot \operatorname{Ln} P_{t}$

que é a clássica versão log-linear da curva de aprendizado, onde LnA e $\mathbf{k}$ são os parâmetros do modelo linear, que podem ser estimados por mínimos quadrados.

Uma dada curva de aprendizado estará completamente caracterizada pelo parâmetro A e a constante $\mathbf{k}$, os quais são determinados através de um ajuste da curva aos dados experimentais. $\mathrm{O}$ valor de A pode variar amplamente, dependendo da natureza da aplicação. A magnitude de $\mathbf{k}$, por outro lado, tem uma faixa de variação muito mais estreita, com curvas de aplicações similares tendendo a valores comuns de $\mathbf{k}$.

Sendo $\mathbf{C}_{\mathbf{t}}$ um custo específico, o valor de $\mathbf{k}$ que denota melhoria deve ser negativo. Um valor positivo de $\mathbf{k}$ indica produtividade deteriorada com custos crescentes, geralmente detectada em processos que estão no início e/ou em ajuste. Se k=0, então não há melhoria na performance do custo com a continuidade da produção.

O modelo log-linear padrão apresentado pode ser usado de diversas formas e variações, de acordo com o problema em análise. Uma versão mais adequada da curva de aprendizado para os dados que serão estimados nesse trabalho é a curva de Stanford-B. Esse modelo foi desenvolvido a partir da observação de que novos programas ou novas condições de trabalho tendem a ser afetados pela experiência ganha durante períodos de produção prévios. Esse efeito de "carry-over" é traduzido na curva loglinear através da adição de um novo parâmetro $\mathbf{p}_{0}$ ao nível de produção. Assim, teremos a eq.(6):

$\mathbf{C}_{\mathrm{t}}=\mathbf{A}\left(\mathbf{P}_{\mathrm{t}}+\mathbf{p}_{0}\right)^{\mathrm{k}}$

O significado físico de $\mathbf{p}_{0}$ é uma quantidade acumulada de produção correspondente às fases anteriores ao novo programa de produção da empresa. A generalização da curva de Stanford-B é obtida quanto se considera não só o parâmetro $\mathbf{p}_{0}$, mas também um novo parâmetro $\mathbf{c}_{\boldsymbol{0}}$, cujo significado é o custo específico acumulado correspondente às fases anteriores ao novo programa de produção da empresa, que é o modelo adotado nesse trabalho conforme indica a eq.(7):

$\left(\mathbf{C}_{\mathrm{t}}+\mathbf{c}_{0}\right)=\mathbf{A}\left(\mathbf{P}_{\mathrm{t}}+\mathbf{p}_{0}\right)^{\mathrm{k}}$

\section{Aplicação do modelo de previsão do custo}

A produção de minério acumulada, em toneladas métricas naturais (TMN), obteve uma boa correlação com os custos $\left(R^{2}=0,98\right)$. A Figura 1 apresenta os dados e a evolução dos custos no período. Para aplicar o modelo de Stanford-B generalizado, a série temporal foi dividida em cinco fases de produção, mostradas na Figura 1 pelos trechos AB, BC, 
$\mathrm{CD}, \mathrm{DE}$ e EF, respectivamente. A fase $\mathrm{AB}$ corresponde aos quatro meses do ano de 1997 que antecederam à conclusão do projeto de expansão da SAMAR$\mathrm{CO}$, quando a capacidade de produção de pelotas foi duplicada de 6 para $12 \mathrm{mi}$ lhões de toneladas anuais, com a construção do segundo forno de pelotização. $\mathrm{O}$ perfil descendente de produção desse trecho $\mathrm{AB}$ pode ser explicado pelas diversas intervenções que se efetuavam nos processos das usinas de concentração e pelotização, devido aos ajustes finais dos novos equipamentos da expansão. A fase BC corresponde ao período pós-expansão, com um gradual aumento na produção de minério, até o patamar médio de 2 milhões de toneladas mensais. Foi uma fase de adaptação às novas metas de produção. O brusco perfil descendente do trecho $\mathrm{CD}$ se deve à retração da demanda de pelotas no mercado asiático, o que obrigou a empresa a reduzir sua produção.A recuperação só veio em $\mathrm{DE}$, quando houve o reaquecimento do mercado de pelotas, além da desvalorização do real frente ao dólar. A combinação desses dois fatores levou a um aumento da produção com a redução de custos. O trecho EF foi individualizado devido a um gasto indireto adicional ocorrido em junho de 2000, na área de serviços contratados.

A Figura 2 fornece as curvas de aprendizado para os cinco trechos descritos anteriormente, através da relação entre o custo específico acumulado e a produção acumulada, na escala logarítmica. Para cada um dos trechos, foram ajustadas retas de tendência que permitiram estimar os parâmetros do modelo de Stanford-B generalizado. Na Figura 2 , os valores de $\mathbf{y}$ e $\mathbf{x}$ correspondem a $\operatorname{Ln}\left(\mathbf{C}_{t}+\mathbf{C}_{\mathbf{o}}\right)$ e $\operatorname{Ln}\left(\mathbf{P}_{\mathbf{t}}+\mathbf{P}_{\mathbf{0}}\right)$, respectivamente.

A justificativa para divisão da série temporal em fases se explica pela necessidade de revalidar o modelo de previsão a cada momento em que se julgue que houve uma mudança significativa no processo. As previsões futuras dos custos têm de levar em consideração todos os possíveis ganhos de produtividade e/ou melhorias, caso contrário os ajustes dos modelos apresentarão irre- gularidades e produzirão estimativas bastante distantes da realidade.

A Tabela 1 resume os parâmetros do modelo de Stanford-B generalizado para cada um dos trechos analisados. Nessa tabela, os valores dos parâmetros A dos modelos ajustados são iguais aos antilogaritmos neperianos dos coeficientes lineares das retas de tendência de cada trecho. Já os valores dos parâmetros $\mathbf{k}$ são iguais aos coeficientes angu- lares dessas retas. Os valores de $\mathbf{C}_{\mathbf{0}}$ e $\mathbf{P}_{\mathbf{0}}$ correspondem aos valores de custo específico acumulado e produção acumulada, respectivamente, do mês imediatamente anterior ao início de cada fase ou trecho em análise. A partir desses parâmetros, determinam-se os índices de aprendizado (IA) e de progresso (IPR).

A Figura 3 apresenta um diagrama de dispersão entre os valores de custos reais e estimados pelos modelos obtidos.

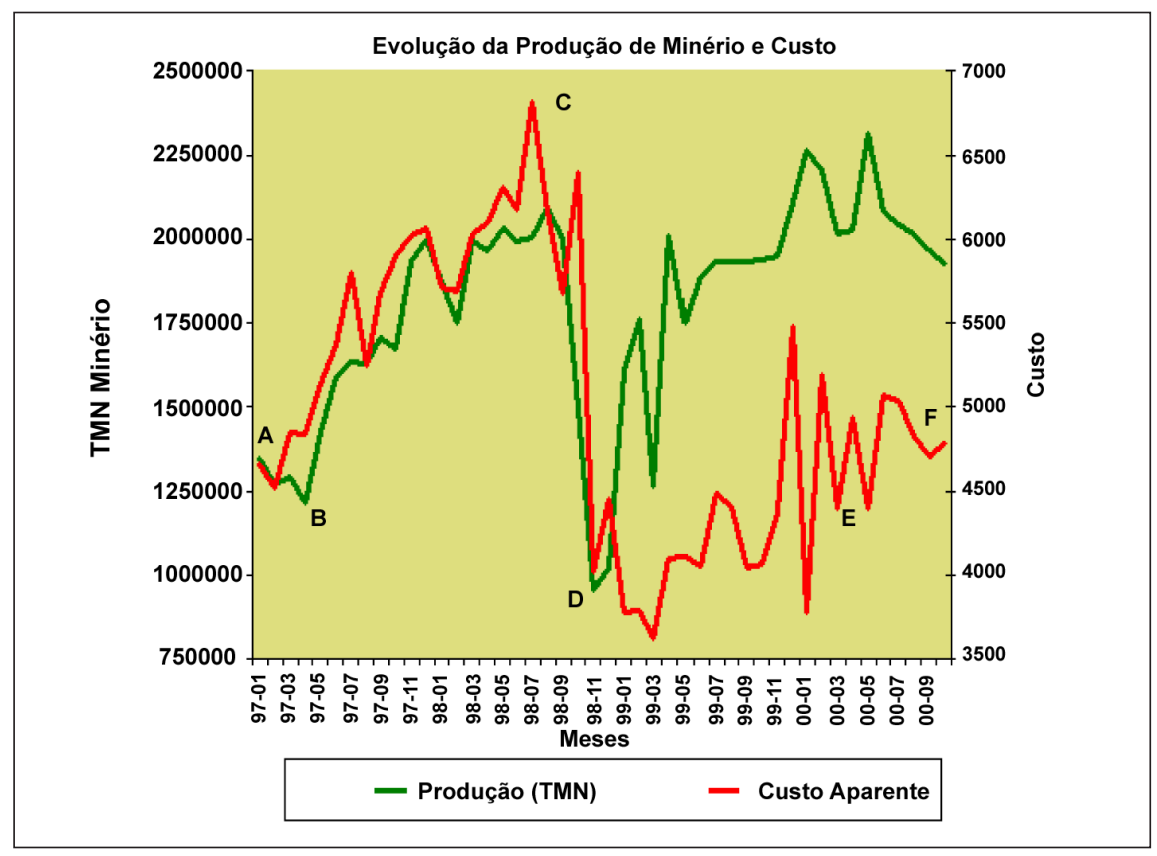

Figura 1 - Evolução da produção de minério e do custo.

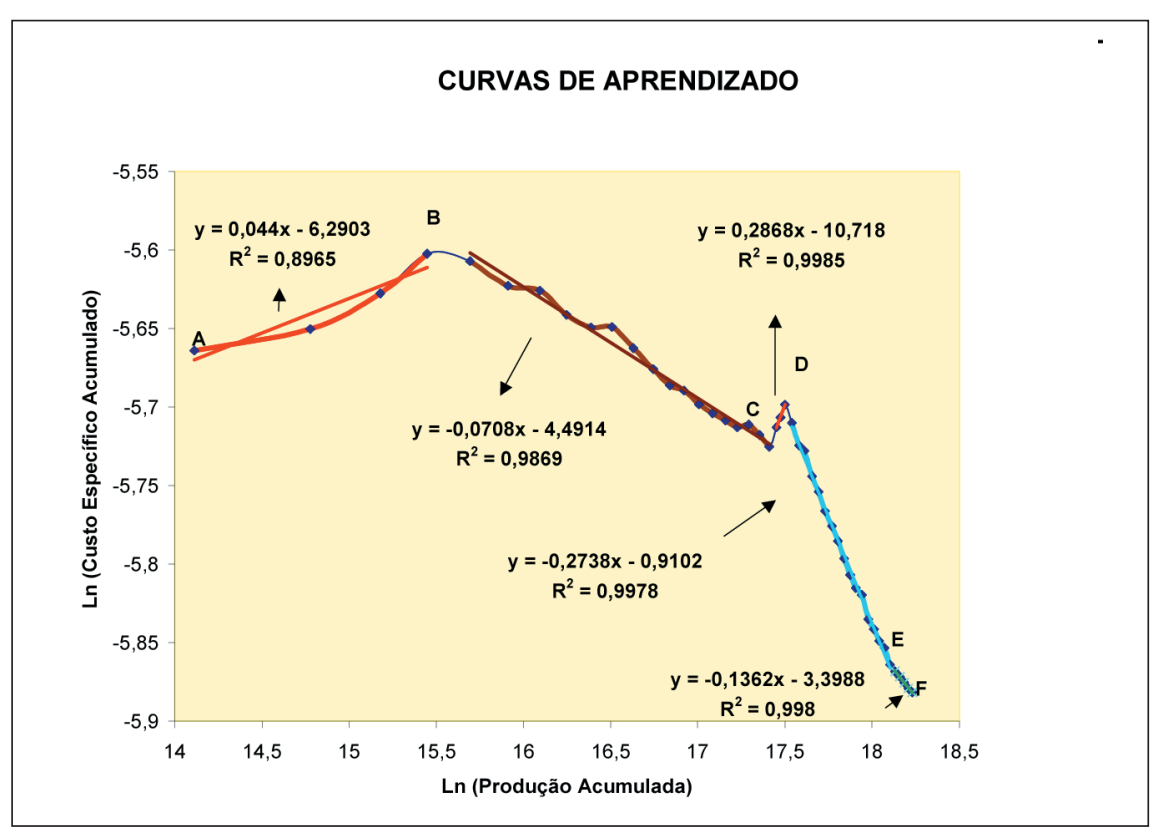

Figura 2 - Modelo de aprendizado ajustado:Relação In (custo acumulado) vs In (produção acumulada). 
Tabela 1 - Parâmetros dos modelos ajustados.

\begin{tabular}{c|c|c|c|c|c|c}
\hline Fase & $\mathbf{C}_{\mathbf{o}}$ & $\mathbf{P}_{\mathbf{o}}(\mathrm{TMN})$ & $\mathbf{A}$ & $\mathbf{k}$ & IA (\%) & IPR (\%) \\
\hline $\mathrm{AB}$ & 0 & 0 & 0,00185 & 0,044 & 103 & -3 \\
\hline $\mathrm{BC}$ & 0,0037 & 5117552 & 0,011 & $-0,0708$ & 95 & 5 \\
\hline $\mathrm{CD}$ & 0,0033 & 36400740 & 0,00002 & 0,2868 & 122 & -22 \\
\hline $\mathrm{DE}$ & 0,0033 & 39876320 & 0,4024 & $-0,2738$ & 83 & 17 \\
\hline $\mathrm{EF}$ & 0,0028 & 72772887 & 0,0334 & $-0,1362$ & 91 & 9 \\
\hline
\end{tabular}

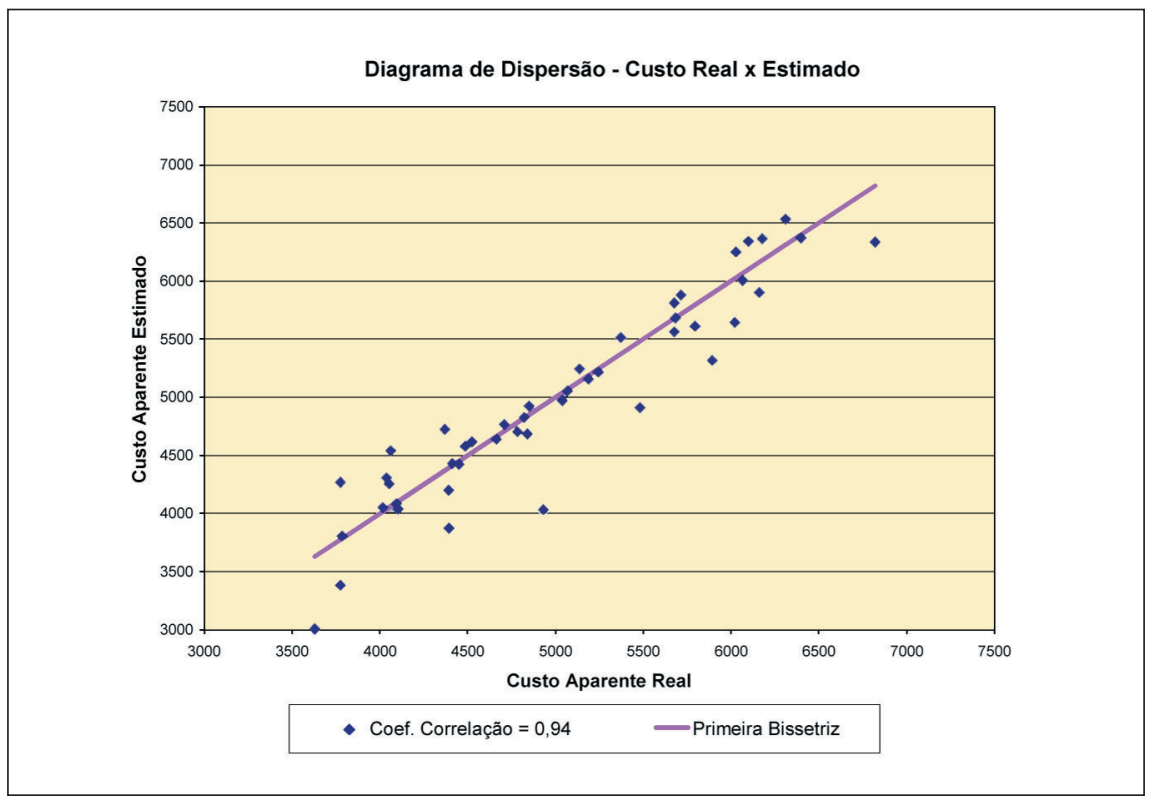

Figura 3 - Diagramas de dispersão: Custo real e estimado.

A magnitude da dispersão pode ser avaliada por:

Dispersão $=E\left[(\text { estimado }- \text { real })^{2} / 2\right]$,

tendo $\mathbf{E}$ o significado de uma esperança matemática.

Determina-se a dispersão relativa:

Dispersão Relativa $=2 *$ Dispersão / Var[( estimado - real) $]$,

tendo Var o significado de variância.

Se a variável [estimado - real] tende a zero (situação esperada), então a sua variância tende ao dobro da dispersão e a dispersão relativa tende a 1 , pois :

Var $[($ estimado - real $)]=E(\text { estimado }- \text { real })^{2}-[E(\text { estimado }- \text { real })]^{2}=2^{*}$ dispersão - [E (estimado - real) $]^{2}$.

A Tabela 2 fornece, além da dispersão relativa, a estatística dos erros relativos de estimativa individuais, avaliados por [(custo estimado - custo real) / (custo real)] $\mathrm{x} 100 \%$.

A Figura 4 fornece uma comparação entre os valores de custos reais e estimados, bem como a previsão do modelo para os cinco meses subseqüentes, conside- rando-se não haver mudança significativa nos processos de produção ou gastos extraordinários. A produção prevista foi fornecida pela equipe de planejamento de curto prazo da SAMARCO e os resultados foram bastante satisfatórios com os valores previstos pelo sistema de aprendizado, situando-se dentro da faixa-tolerância próxima de $10 \%$ dos custos operacionais da mina.

\section{Conclusões}

As curvas de aprendizado utilizadas apresentaram uma boa conformidade com o modelo de Stanfordb. Foram feitas as reavaliações necessárias ao longo da série temporal, permitindo que os modelos ajustados assimilem as mudanças de estado ocorridas no processo, pois as previsões da performance do custo se fundamentam na manutenção de determinadas condições de trabalho.

Como medida de precisão efetiva na aplicação do método, os resultados obtidos permitiram indicar erros relativos de estimativa entre 10 e $+10 \%$ com $85 \%$ de certeza.

Deve-se estar atento ao problema da estabilidade dos parâmetros dos modelos ajustados nesse trabalho, pois a estrutura estatística dos mesmos advém do uso da variável dependente produção de minério, que pode apresentar valores consideráveis de autocorrelação. Esse fato pode levar a um viés local na previsão de custo, mesmo quando os desvios relativos médios são baixos quando se analisa a série temporal globalmente.

A metodologia da curva de aprendizado, se adequadamente implementada, pode vir a ser útil nas atividades gerenciais, tais como planejamento, orçamento, estabelecimento de metas e análise econômica. Trata-se de uma ferramenta analítica auxiliar na tomada de decisões, permitindo que as melhorias de produtividade e de desempenho do custo possam ser previsíveis e racionalizadas. 
Tabela 2 - Avaliação dos erros relativos de estimativa.

\begin{tabular}{l|c}
\hline Custo Médio Real & 5023 \\
\hline Custo Médio Estimado & 4976 \\
\hline Média dos Erros Relativos & $-0,86 \%$ \\
\hline Média dos Valores Absolutos dos Erros Relativos & $4,22 \%$ \\
\hline Freqüência de Erros Relativos entre $-10 \%$ e $+10 \%$ & $85 \%$ \\
\hline Dispersão Relativa & 1.006 \\
\hline
\end{tabular}

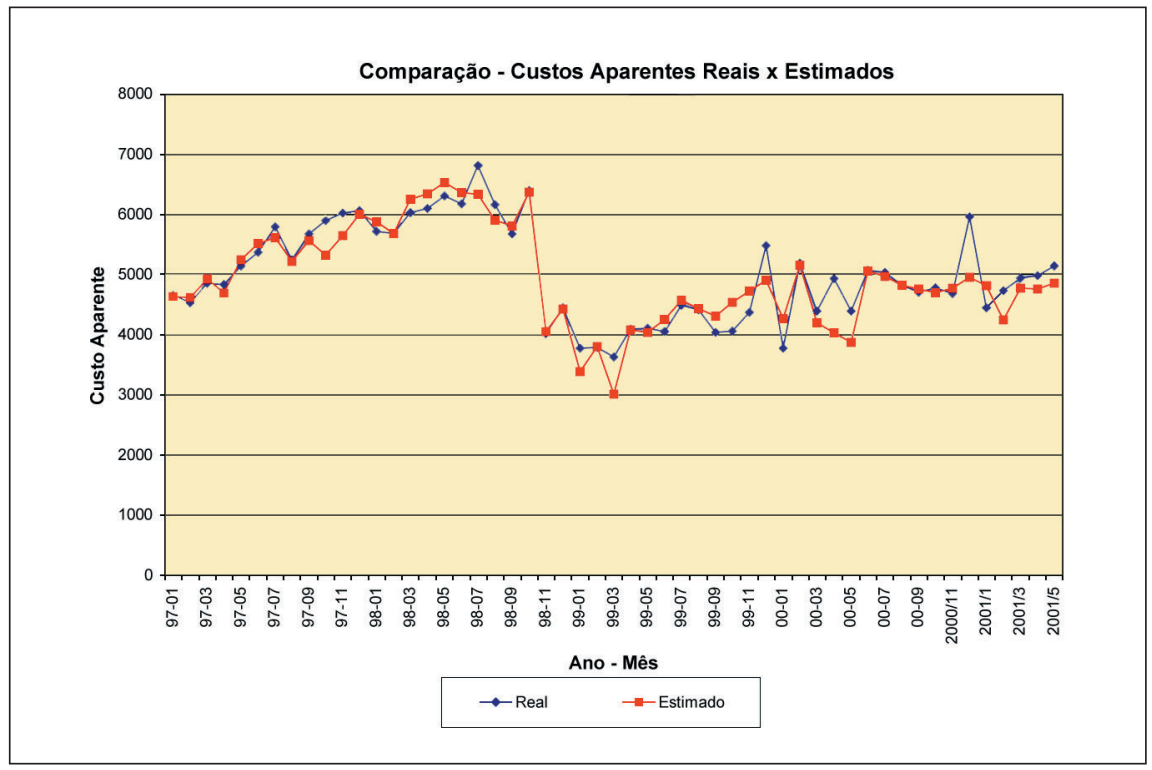

Figura 4 - Comparação entre os custos reais e os estimados pelo modelo Stanford-B.

\section{Agradecimentos}

Os autores gostariam de agradecer o apoio da SAMARCO S/A pela cessão das informações e do CNPq para a realização desse trabalho.

\section{Referências} bibliográficas

BELKAOUI,A. The learning curve: a management accounting tool. London: Quorum Books, 1986. 245p. Boston Consulting Group. Perspectives on experience. Boston: HMSO, 1970. $109 \mathrm{p}$.

PIERSON, G. Learning curves make productivity gains predictable. Engineering \& Mining Journal. August 1981 .

SILVA, L. G. O, SUSLICK, S. B. Desenvolvimento de um modelo de previsão para o consumo de estanho no Brasil. Cadernos IG/UNICAMP, v. 3 , n. 1, p.23-29, 1993

SUSLICK, S. B., HARRIS, D. P. LongRange consumption forecasts using innovative mehtods: the case of aluminum in Brazil to the year 2000 . Resources Policy. v.16, n.3, 1990.

TOWILL, D. R. Forecasting learning cuves. International Journal of Forecasting, v. 6, n. 1, p. 25-38, 1990.

Artigo recebido em 19/03/2003 e aprovado em 10/06/2003.

\section{Em janeiro de 2003 a REM completou 67 anos. REM 67 anos divulgando ciência.}

www.rem.com.br ww.scielo.br 